\title{
DESCRIPCIONES DE DOS ESPECIES NUEVAS DE ENCARSIA FÖRSTER (HYMENOPTERA: APHELINIDAE) Y NUEVOS REGISTROS DE ESPECIES DE ESTE GÉNERO PARA CHIAPAS Y JALISCO, MÉXICO
}

\author{
Myartseva S.N.1 y A. GoNZÁLEZ-HERNÁNDEZ ${ }^{2}$ \\ 1 División de Estudios de Postgrado e Investigación, UAM Agronomía y Ciencias, \\ Universidad Autónoma de Tamaulipas, Cd. Victoria, 87149, Tamaulipas, MÉXICO. \\ 2 Facultad de Ciencias Biológicas, Universidad Autónoma de Nuevo León, \\ San Nicolás de Los Garza, Monterrey, Nuevo León, MÉXICO. \\ smyartse@uat.edu.mx; agonzale@fcb.uanl.mx
}

\section{RESUMEN}

Se describen e ilustran dos nuevas especies del género Encarsia Förster, colectadas en Chiapas y Jalisco: Encarsia clavata sp.n. y Encarsia verticina sp.n.. Nueve registros nuevos de las especies de este género son presentadas para los estados de Chiapas y Jalisco, incluyendo dos registros nuevos para México.

Palabras clave: Aphelinidae, Encarsia, especies nuevas, Chiapas, Jalisco, México.

\begin{abstract}
Two new species of genus Encarsia Förster collected in Chiapas and Jalisco are described and illustrated: Encarsia clavata sp.n. and Encarsia verticina sp.n.. Nine new records of species of this genus are given for the states of Chiapas and Jalisco, including two new records for Mexico.

Key words: Aphelinidae, Encarsia, new species, Chiapas, Jalisco, Mexico.
\end{abstract}

\section{INTRODUCCIÓN}

Encarsia Förster, 1878, es uno de los géneros más ricos en especies de la familia Aphelinidae. Este género cosmopolita contiene actualmente cerca de 280 especies descritas, divididas en más de 20 grupos de especies (de acuerdo a Hayat 1998). La primera lista de referencias de Aphelinidae de México incluyó 30 especies del género Encarsia (Myartseva y Ruíz Cancino 2000) y el último registro de Encarsia de México incluyó 38 especies (Myartseva et al. 2004), pero estas listas pueden ser incrementadas al doble después de la identificación y descripción de muchas nuevas especies para la ciencia y para México, las cuales son preservadas en las colecciones 
entomológicas de las universidades de los estados de Tamaulipas y Nuevo León, entre otros.

Las especies de Encarsia son parasitoides primarios y tienen gran importancia económica para el control biológico de plagas en muchos países del mundo y para el control natural de escamas armadas y mosquitas blancas (Homoptera: Coccoidea, Aleyrodoidea) en diferentes áreas ambientales. Varias especies son explotadas en programas de introducción de insectos benéficos para el control biológico de plagas agrícolas. En México fueron introducidos parasitoides de mosquitas blancas y escamas armadas. Algunas especies de Encarsia están siendo utilizadas exitosamente contra insectos homópteros plagas: Encarsia perplexa Huang y Polaszek, E. aurantii (Howard), E. formosa Gahan y E. clypealis (Silvestri) (Myartseva y Ruíz Cancino 2000).

El género Encarsia está ampliamente distribuido en México. Para el estado de Jalisco son conocidos únicamente datos de cinco especies introducidas en México para el control biológico de la mosca prieta de los cítricos Aleurocanthus woglumi Ashby: E. clypealis (Silvestri), E. divergens (Silvestri), E. merceti Silvestri, E. opulenta (Silvestri) y E. smithi (Silvestri) (Myartseva y Ruíz Cancino 2000). Por el contrario, no existen hasta ahora datos sobre especies de Encarsia para el estado de Chiapas. Varias nuevas especies de diferentes estados de la República Mexicana fueron colectadas y descritas en el período de 1997 al 2006.

Los datos sobre once especies de Encarsia distribuidas en los estados de Chiapas y Jalisco son presentados por primera vez en este artículo: dos especies son nuevas para la ciencia, nueve especies son registradas por primera vez en dichos estados, siendo dos de éstas últimas nuevos registros para México.

\section{MATERIALES Y MÉTODOS}

Los especímenes de Encarsia estudiados taxonómicamente fueron colectados en los estados de Chiapas y Jalisco, y se encontraban depositados en las colecciones entomológicas de la Universidad de Tamaulipas, UAM Agronomía y Ciencias, Cd. Victoria, y la Universidad de Nuevo León, Facultad de Ciencias Biológicas, San Nicolás de los Garza. Los especímenes de Encarsia fueron criados de hospederos y también recolectados en los años de 1997, 2000 y 2006, utilizando trampas Malaise, platos amarillos con agua y red de golpeo con malla. Todos los especímenes fueron preservados en alcohol al 75\%. Para la determinación se prepararon laminillas con bálsamo de Canadá siguiendo los métodos tradicionales para avispas calcidoideas (Noyes 1982). Se utilizaron varias revisiones, claves para la determinación de especies de Encarsia, descripciones originales y la colección de referencia de afelínidos de la UAT (Hayat 1989, 1998; Polaszek et al. 1992; Schauff et al. 1996; Huang y Polaszek 1998). 
Abreviaturas para instituciones depositarias del material examinado: BNMH Natural History Museum, London, U.K.; USNM - National Museum of Natural History, Washington, D.C., EUA; UANL - Universidad Autónoma de Nuevo León, Monterrey, México; CIB - Colección de Insectos Benéficos de la Universidad Autónoma de Nuevo León, Monterrey, México; UAT - Universidad Autónoma de Tamaulipas, Ciudad Victoria, México.

\section{RESULTADOS}

\section{Descripciones de las especies nuevas}

\section{Encarsia clavata Myartseva y González, sp. n. \\ Figuras 1-5}

Hembra. Longitud del cuerpo: 0.72-0.88 mm (Holotipo $-0.75 \mathrm{~mm}$ ).

Coloración. Cabeza amarilla, fronto-vértice pálido, mejillas de color pardo en la mitad baja, área triangular detrás del ocelo posterior ligeramente oscuro, occipucio pardo bajo el foramen, antena amarilla blanquecina, escapo y segmento apical de la clava ligeramente oscuro. Mesosoma pardo, la parte posterior del lóbulo medio y lóbulos laterales del mesoescudo, escutelo y metanoto de color amarillo. Alas hialinas. Patas blanquecinas, fémur y tibia ligeramente oscura cerca de la base. Metasoma pardo, séptimo terguito amarillo blanquecino, ovipositor amarillo, la tercera válvula y porción lateral del valvífero y el ápice de los estiletes color pardo negruzco.

Morfología. Cabeza tan ancha como el mesosoma y 1.3-1.4 veces tan ancha como alta. Fronto-vértice transversalmente estriado, 0.6 veces tan ancho como la anchura de la cabeza. Ocelos formando un pequeño triángulo rectángulo, ocelos posteriores separados del margen de los ojos por una distancia aproximada de 3-4 diámetros ocelares. Ojos aproximadamente 1.4 veces tan largos como las mejillas. Mandíbulas tridentadas. Sutura malar presente. Escrobes antenales profundos y unidos arriba. Antenas (Fig. 1) insertadas cerca del margen de la boca; distancia entre tórulos de 0.7-0.8 veces tan larga como la distancia del tórulo al margen del ojo. Escapo cerca de 4 veces tan largo como ancho; pedicelo cerca de dos veces tan largo como ancho y más corto que los primeros dos segmentos funiculares combinados; todos los segmentos del flagelo más largos que anchos; primer segmento funicular de 1.6-1.9 veces más largo que ancho; segundo segmento ligeramente más largo y ancho, también 1.61.9 veces tan largo como ancho (una hembra con segundo segmento 2.1 veces tan largo como ancho); clava con cuatro segmentos, ligeramente más corta que el funículo, pedicelo y escapo combinados; del segundo al quinto segmento flagelar cada uno con una sensila. Mesosoma con la superficie reticulada. El lóbulo medio del mesoescudo (Fig. 2) con 4-5 pares de sedas (una hembra con tres pares de sedas), lóbulos laterales cada uno con dos sedas, cada axila con una seda. Lóbulo medio cerca de 1.8 veces tan ancho como largo. Escutelo de 0.6 veces tan 
largo como el lóbulo medio y cerca de dos veces tan ancho como largo. Sensila placoidal en el escutelo ampliamente distribuida. Distancia entre el par posterior de sedas cerca de 0.7 veces tan larga como entre el par anterior de sedas. Ala frontal sin una área asetosa cercana a la vena estigmal, cerca de 2.5 veces tan larga como ancha, su fleco marginal de $0.2-0.3$ veces tan largo como el ancho máximo de ala. Vena marginal con 5-6 sedas largas sobre el margen anterior y ligeramente más larga que la vena submarginal; vena estigmal muy corta y cercana al margen del ala (Fig. 3). Base del ala con 3-4 sedas sobre el disco. Ala posterior cerca de 5.3 veces tan larga como ancha, su fleco marginal ligeramente más corto que el máximo ancho del ala. Fórmula tarsal 5-5-5. Espuela de la tibia media (Fig. 4) cerca de 0.8 veces tan larga como el basitarso; basitarso casi igual en longitud a los siguientes dos segmentos tarsales combinados. Los terguitos segundo al séptimo del metasoma con 2, 2, 2, 6, 6, y 4 sedas, respectivamente. Ovipositor (Fig. 5) con base al nivel del tercer terguito, ligeramente sobresaliente, cerca de 1.5 veces tan largo como la tibia media. Tercer válvula 0.5 veces tan larga como el segundo valvífero.

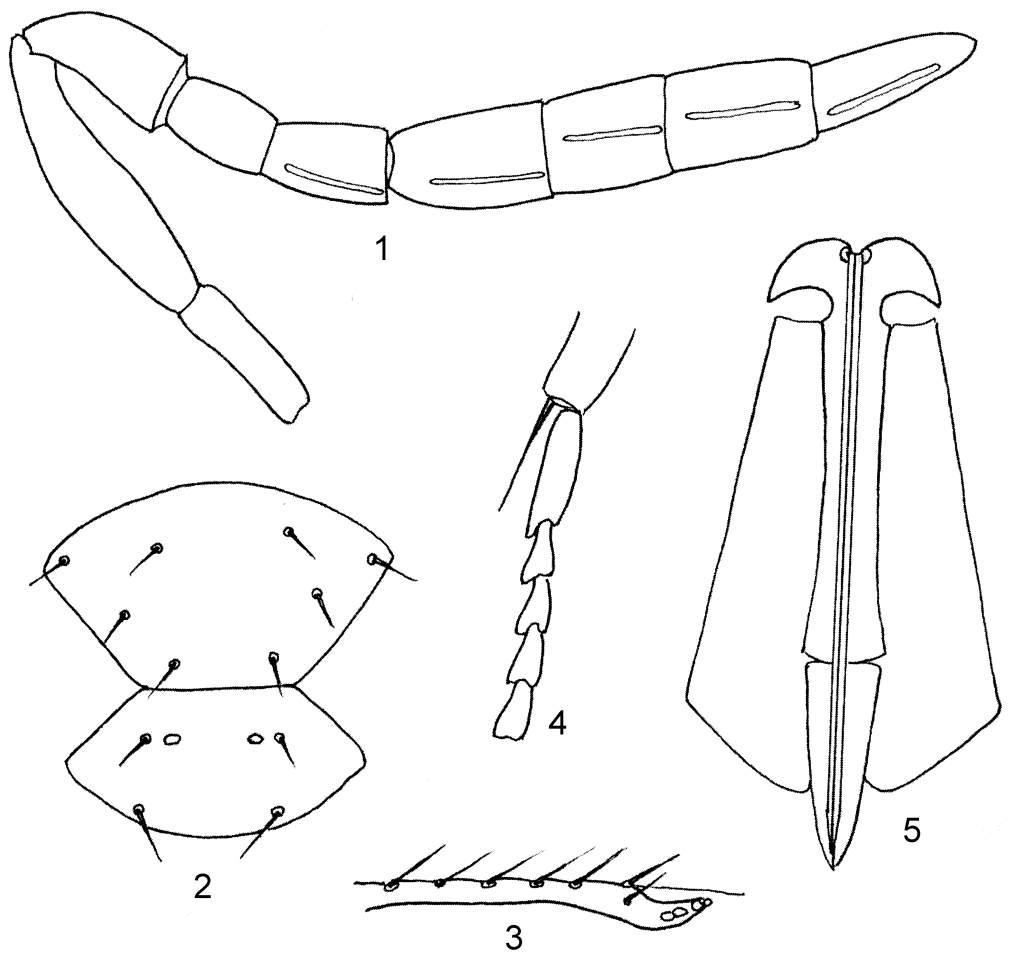

Figs 1-5. Encarsia clavata, sp.n.: 1 - antena, hembra, 2 - el lóbulo medio del mesoescudo y escutelo, 3 - venas estigmal y marginal del ala anterior, 4 - espuela de la tibia media y tarso, 5 - ovipositor. 
Macho. Desconocido.

Comentarios. La antena con clava de 4 segmentos es muy rara en Encarsia (por ejemplo, $E$. nanjingensis Hayat y Polaszek), también la clava no clavada o no distintamente clavada ( $E$. smithi Silvestri, E. sueloderi Polaszek, E. phaea Huang y Polaszek). En la fauna mexicana de Aphelinidae, E. clavata sp. n. es la primera especie nativa con clava de cuatro segmentos. Puede distinguirse de E. nanjingensis por lo siguiente: en E. nanjingensis - el lóbulo medio del mesoescudo con tres pares de sedas, ala anterior oscura bajo la vena marginal y cerca de tres veces tan largo como ancho, su fleco marginal de 0.63 veces tan largo como el ancho del ala, tercer válvula de 0.42 veces tan larga como el segundo valvífero, base del ovipositor al nivel del primer terguito, segundo segmento funicular sin sensila; en E. clavata - el lóbulo medio del mesoescudo con 4-5 pares de sedas, ala anterior hialina y 2.5 veces tan largo como ancho, su fleco marginal de 0.2-0.3 veces tan largo como el ancho de la ala, tercer válvula de 0.5 veces tan larga como el segundo valvífero, base del ovipositor al nivel del tercer terguito, el segundo segmento funicular con sensila.

Encarsia smithi, que fuera introducida en México hace más de 50 años para el control biológico de Aleurocanthus woglumi Ashby (Jiménez-Jiménez y Smith 1958), no tiene una antena clavada distinguible, el ala anterior es oscura bajo la vena marginal, el ovipositor es más corto que la tibia media y basitarsos combinados, la tercer válvula de 0.41 veces tan larga como el segundo valvífero y el segundo segmento funicular dos veces tan largo como el primer segmento. En E. clavata - la antena es distinguiblemente de cuatro segmentos, ala anterior hialina, el ovipositor 1.5 veces tan largo como la tibia media, tercer válvula de 0.5 veces tan larga como el segundo valvífero y el segundo segmento funicular de 1.1-1.3 veces tan largo como el primer segmento.

Material examinado. Holotipo hembra. México: Chiapas - Jiménez Ceballos, redeo, Hosp. 3,12, 12.VIII.2000 (col. A. González H.), CIB 00-0090. Paratipos - datos iguales que el holotipo, 1 hembra; Jaltenango, Reserva El Triunfo, Mirador Santa Rita, red de golpeo con malla (22), 22.VII.1997, 1 hembra (col. A. González H.). Jalisco - La Huerta, Est. Biol. Chamela UNAM, Vereda Tejón, redeo en maleza, $162 \mathrm{msnm}, \mathrm{N} 19^{\circ} 30.559^{\prime \prime}$, W $105^{\circ}$ 02.464" , 27.VII.2000, 1 hembra (col. Y. Castillo O., A. Dávila L.y A. González H.), CIB 000039; Vereda Tejon-Ardilla, t. Malaise 2, en maleza, 17-21.I.2000, 1 hembra (col. M.A. Sarmiento), CIB 00-0065; Camino Chachalaca, redeo en maleza, 96.5 msnm, N 19²9.791" , W $105^{\circ} 02.553^{\prime \prime}$, 26.VII.2000, 1 hembra (col. Y. Castillo O., A. Dávila L.y A. González H.), CIB 00-0036.

Localidad tipo: México, Chiapas, Jiménez Ceballos.

Depositarios del material tipo: Entomological Research Museum of the Department of Entomology, University of California, Riverside, California, USA. Paratipos preservados: 1 hembra en el mismo museo; 2 hembras en el USNM, 1 hembra en el BMNH, 1 hembra en la UANL. 


\section{Encarsia verticina Myartseva y González, sp.n. Figuras 611}

Hembra. Longitud del cuerpo: 0.53-0.63 mm.

Coloración. Cabeza amarilla clara, occipucio entre el foramen y los ojos y fronto-vértice ligeramente oscurecido, clípeo, mejillas y una banda delgada transversal detrás del ocelo posterior en el fronto-vértice de color pardo, antena blanquecina. Mesosoma amarillo claro, pronoto, margen anterior del mesoescudo pardo, el lóbulo medio del mesoescudo sobre la parte media y propodeo oscurecido. Alas oscurecidas en la parte media basal, margen posterior de la vena marginal parduzco. Patas blanquecinas, fémur posterior ligeramente oscurecido. Metasoma pardo, séptimo terguito y ovipositor blanquecino.

Morfología. Cabeza tan ancha como el mesosoma y cerca de 1.3 veces tan ancha como su altura. Fronto-vértice cerca de 0.5 del ancho de la cabeza. Ocelos formando un pequeño triángulo rectángulo; ocelos posteriores separados del margen de los ojos por una distancia aproximada de 2.5 veces más larga que el diámetro ocelar. Ojos cerca de 1.6 veces tan largos como las mejillas. Mandíbula con tres pequeños dientes no muy claros. Sutura malar presente. Escrobos antenales superficiales. Antenas (Fig. 6) insertadas cerca de el margen de la boca. Distancia entre tórulos aproximadamente tan larga como la distancia del tórulo al margen del ojo. Escapo cerca de 4 veces tan largo como ancho. Pedicelo 2.5 veces tan largo como ancho y cerca de 1.5 veces más largo que el primer segmento funicular. El primer segmento funicular cerca de dos veces tan largo como ancho; segundo segmento cerca de 2.6 veces más largo que ancho, tercero y cuatro segmentos cada uno cerca de tres veces tan largos como anchos. Clava con dos segmentos, ligeramente fusionados, apenas más ancha que el funículo y ligeramente más larga que los dos segmentos funiculares precedentes combinados. Primero y segundo segmentos flagelares sin sensila linear, tercer segmento con una sensila, cuarto al sexto segmento cada uno con dos sensilas. El lóbulo medio del mesoescudo (Fig. 7) con la superficie ampliamente reticulada y con dos pares de sedas, lóbulos laterales cada uno con dos sedas, cada axila con una seda. Lóbulo medio cerca de 1.4 veces tan ancho como largo. Escutelo 0.7 veces tan largo como el lóbulo medio y cerca de dos veces tan ancho como largo. Sensila placoidal en el escutelo ampliamente distribuida, separada por una distancia de cerca de 4 diámetros de sensila. La distancia entre el par de sedas anteriores y entre el par de sedas posteriores es similar. Ala anterior con una área asetosa cercana a la vena estigmal, y cerca de 3.5 veces tan largo como ancho, su fleco marginal cerca de 0.8 veces tan largo como el ancho máximo del ala, base con dos setas. Vena marginal (Fig. 8) con 6 sedas largas sobre el margen anterior y casi igual en longitud a la vena submarginal. La vena estigmal corta y casi sésil. Ala posterior cerca de 10 veces tan larga como ancha, su fleco marginal cerca de 2.5 veces tan largo como el máxima anchura del ala. Formula tarsal 5-5-5. Espuela de la tibia media (Fig. 9) cerca de 0.8 veces tan larga como el basitarso; basitarso tan largo como los siguientes dos segmentos tarsales combinados. Los terguitos segundo al séptimo del metasoma con 2, 2, 2, 4, 2, y 4 sedas respectivamente. Ovipositor (Fig. 10) con base al nivel del cuarto terguito, no sobresaliente, cerca de 0.9 veces tan largo como la tibia media. Tercer válvula de 0.6 veces tan larga como el segundo valvífero y con pequeñas excavaciones dentro del ápice de cada válvula (en todas las hembras). 
Macho. Longitud del cuerpo: $0.53 \mathrm{~mm}$.

Coloración. Coloración parecida a la hembra.

Morfología. Fronto-vértice de 0.6 veces tan largo como el ancho de la cabeza. Ojos cerca de 1.4 veces tan largos como las mejillas. Antenas (Fig. 11) insertadas inmediatamente arriba al nivel inferior del margen de los ojos. Escapo 3.3 veces tan largo como ancho; pedicelo 1.7 veces tan largo como ancho. El primer segmento funicular tres veces tan largo como ancho, cada uno de los segmentos del segundo al cuarto de 3.5 veces tan largo como ancho; segmentos de clava ligeramente fusionados; clava más corta que dos segmentos funiculares precedentes combinados. Todos los segmentos flagelares con dos sensilas. Ala anterior con fleco marginal tan larga como el anchura máxima del ala. Ala posterior 12.5 veces tan larga como ancha, su fleco marginal 3 veces tan largo como la máxima anchura del ala. Genitalia ligeramente más larga que la tibia media.
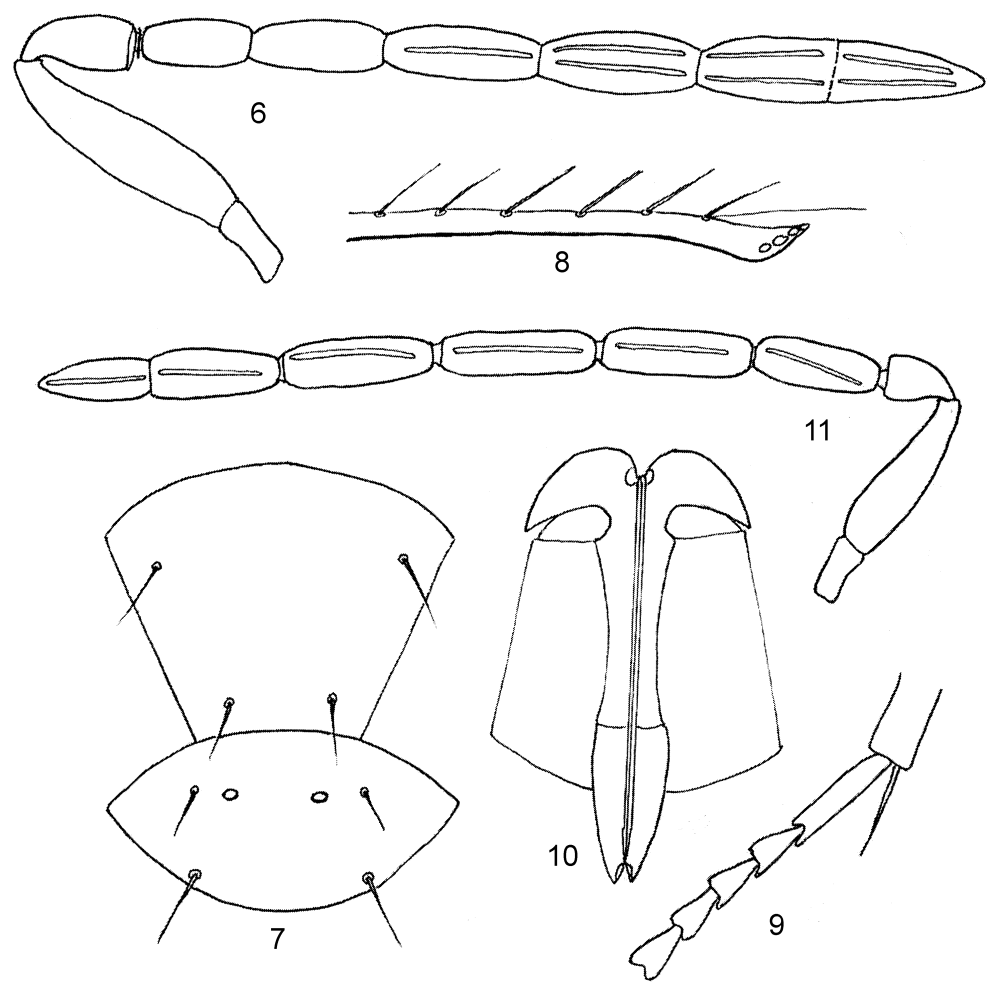

Figs 6-11. Encarsia verticina, sp.n.: 6 - antena, hembra, 7 - el lóbulo medio del mesoescudo y escutelo, 8 - venas estigmal y marginal del ala anterior, 9 - espuela de la tibia media y tarso, 10 - ovipositor, 11 - antena, macho. 
Comentarios. De acuerdo a la clave de Hayat sobre los grupos de especies de Encarsia (Hayat 1998), E. verticina sp. n. pertenece al grupo de especies parvella, pero difiere de todas las especies de este grupo por la tercer válvula, la cual tiene ápice redondeado con una pequeña excavación en el lado interno. En este grupo la coloración similar del metasoma (completamente pardo oscuro, excluyendo el séptimo terguito) lo tiene únicamente $E$. sueloderi Polaszek, descrita de Costa Rica (Mound et al. 1994). Pero Encarsia verticina puede distinguirse de E. sueloderi por lo siguiente: en E. sueloderi la cabeza es uniformemente pardo, el lóbulo medio del mesoescudo con 4 pares de sedas, antena con clava no clavada, ovipositor tan largo como la tibia media; en E. verticina - la cabeza es amarilla, el lóbulo medio del mesoescudo con dos pares de sedas, la clava antenal con dos segmentos, ovipositor más corto que la tibia media $(0.9 \mathrm{X})$.

Material examinado. Holotipo hembra - México, Chiapas, Reserva El Triunfo, sendero Palo

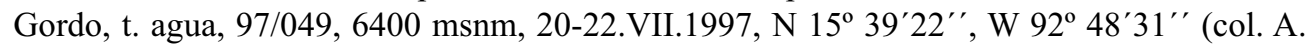
González Hdz., J.B. Woolley, L. Montoya), CIB B97-0.57. Paratipos - datos similares que el holotipo, 5 hembras, 2 machos. Chiapas, Reserva El Triunfo, Mirador Santa Rita, red de golpeo, N 15³9'34', W 92 48'28', 2.VII.1997, 1 macho (col. A. González Hdz.), CIB 97061; Jaltenango, Reserva El Triunfo, Mirador Santa Rita, red de golpeo con malla (22), 22.VII.1997, 2 hembras (col. A. Hernández G.).

Localidad tipo: México, Chiapas, Reserva El Triunfo, sendero Palo Gordo.

Depositarios del material tipo: Entomological Research Museum of the Department of Entomology, University of California, Riverside, California, EUA. Paratipos preservados: 2 hembras y 1 macho en el mismo Museo; 2 hembras y 1 macho en el USNM, 2 hembras y 1 macho en el BMNH, 1 hembra en la UANL.

\section{Comentarios de los nuevos registros*}

\section{Encarsia aurantii (Howard, 1894)}

Hospederos. Diaspididae, raramente Aleyrodidae, Coccidae y Kermesidae (Noyes 2006). En México fue criado de Chrysomphalus aonidum (L.) (De Santis 1979; Myartseva y Ruíz Cancino 2000).

Distribución. Casi cosmopolita; México - Baja California Sur, Jalisco*, Tamaulipas (colecciones de UANL y UAT).

Material examinado. México: Jalisco, La Huerta, Est. Biol. Chamela UNAM, Camino Eje Central, redeo, maleza, 127 msnm, N $19^{\circ} 30.416^{\prime \prime}$, W 105 02.238", 26.VII.2000, 1 hembra (col. Y. Castillo O., A. Dávila L. y A. González H.), CIB 00-0032.

Comentarios. Encarsia aurantii fue introducida en Texas y California para el control de Chrysomphalus aonidum y Melanaspis obscura y en ambos lugares se establecieron (Dean 1982; Ehler 1997). Esta especie fue introducida en México durante 1949-1950 también en contra de la escama roja de Florida Chrysomphalus aonidum y su liberación fue exitosa (Anónimo 1979). 


\section{Encarsia coquilletti Howard, 1895}

Hospederos. Aleyrodidae.

Distribución. EUA (Schauff et al. 1996; Noyes 2006); México* - Jalisco.

Material examinado. México: Jalisco, La Huerta, Est. Biol. Chamela UNAM, Camino

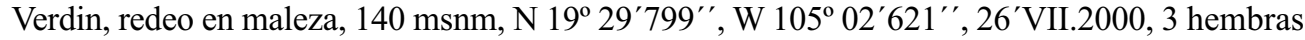
(col. Y. Castillo O., A. Dávila L.y A. González H.), CIB 00-0038.

Comentarios. Nuevo registro para México.

\section{Encarsia costaricensis Evans \& Angulo, 1996}

Hospederos. Aleyrodidae - Trialeurodes vaporariorum (Westwood) (Evans y Angulo, 1996).

Distribución. Costa Rica; Mexico* - Chiapas.

Material examinado. Mexico: Chiapas, Balúm Canal, Km 6 Tenejapa, red de golpeo, 7400


97-047.

Comentarios. Nuevo registro para México.

\section{Encarsia formosa Gahan, 1924}

Hospederos. Aleyrodidae (más de 20 especies). En México fue criado de Trialeurodes vaporariorum (Westwood), Aleurothrixus floccosus (Maskell), Bemisia tabaci-complejo y Aleyrodes sp. (Myartseva et al. 2006; colección de la UAT).

Distribución. Cosmopolita; México - Baja California, Jalisco*, Guerrero, D.F., Tamaulipas (colecciones de la UAT y UANL).

Material examinado. México: Jalisco, La Huerta, Est. Biol. Chamela UNAM, Camino

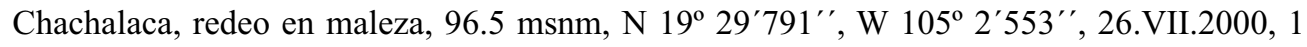
hembra (col. Y. Castillo O., A. Dávila L.y A. González H.), CIB 00-0036.

Comentarios. Poblaciones de Encarsia formosa de Egipto fueron introducidas a Baja California, México en 1992 para el control de Bemisia tabaci y B. argentifolii (Hennessey et al. 1995).

\section{Encarsia haitiensis Dozier, 1932}

Hospederos. Aleyrodidae. En México fue criado de Aleurothrixus floccosus (Alvarado-Mejía y González-Hernández 1990).

Distribución. Benin, Cuba, Haiti, Hawai, Taiwan, Venezuela (Noyes, 2006); México - Jalisco*. 
Material examinado. México: Jalisco, La Huerta, Est. Biol. Chamela UNAM, Camino

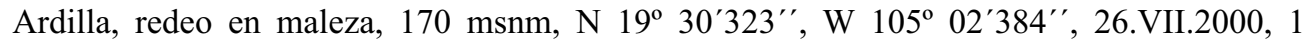
hembra (col. Y. Castillo O.y A. Dávila L.), CIB 00-0034.

Comentarios. De Santis (1989) registró la distribución de Encarsia haitiensis para México sin indicación de localidades.

\section{Encarsia luteola Howard, 1895}

Hospederos. Aleyrodidae. En México fue criado de Bemisia tabaci-complejo, Trialeurodes sp. y Tetraleurodes acaciae (Myartseva y Ruíz Cancino 2000).

Distribución. Brasil, Chile, Cuba, Guadalupe, Puerto Rico, Israel (introducida), Africa de Sur, EUA (Noyes 2006); México - Chiapas*, Colima, Guerrero, Sinaloa, Tamaulipas (colecciones de la UANL y UAT).

Material examinado. México: Chiapas, El Bosque de Aldama, redeo, Alamillo-zacates-


González H.), CIB 00-0087.

Comentarios. Encarsia luteola fue introducida a Israel para el control de Bemisia tabaci y establecida (Rivnay y Gerling 1988).

\section{Encarsia meritoria Gahan, 1927}

Hospederos. Aleyrodidae.

Distribución. Bahamas, Bermuda, Brasil, República Dominicana, Trinidad y Tobago, Francia, Israel, Italia, España, África del Sur, EUA (Noyes 2006); México - Chiapas*.

Material examinado. México: Chiapas, Reserva El Triunfo, sendero Palo Gordo, t. agua, 6400 msnm, 97/049, N 15 39'22', W 92 48'31', 20-22.VII.1997, 1 hembra (col. A. González Hdz., J.B. Woolley y L. Montoya), CIB 97-057; El Encajonado, sendero El Túnel, red de golpeo, 28.VII.1997, 1 hembra (col. A. González Hdz.), CIB 97-070.

Comentarios. Schauff et al. (1996) registró la distribución de Encarsia meritoria para México sin indicación de localidades. Encarsia meritoria fue introducida a Israel para el control de Bemisia tabaci y a Italia en contra de Parabemisia myricae (Argov y Rössler 1988; Barbagallo et al. 1992). Viggiani (1989) sinonimizó E. meritoria con E. hispida. De acuerdo al rechazo de esta especie de Polaszek et al. (1992) y a un estudio molecular de estas especies de Babcock et al. (2001), ambas son especies distintas.

\section{Encarsia perplexa Huang y Polaszek, 1998}

Hospederos. Aleyrodidae. En México fue criado de Aleurocanthus woglumi (JiménezJiménez y Smith 1958). 
Distribución. China, Taiwan, India, EUA; México - Chiapas*, Colima, Jalisco, Morelos, Nuevo León, San Luis Potosí, Sinaloa, Tamaulipas, Veracruz (Noyes 2006; colección de la UAT).

Material examinado. México: Chiapas, Tapachula, ex Aleurocanthus woglumi en Citrus aurantifolia, 27.IX.2006, 1 hembra y 1 macho (col. S. Myartseva).

Comentarios. Encarsia perplexa Huang y Polaszek hasta 1998 fue conocida en México como especie introducida Encarsia opulenta (Silvestri) y su liberación fue exitosa. Ahora esta especie está ampliamente distribuida en estados productores de cítricos (Myartseva y Luna Salas 2005).

\section{Encarsia variegata Howard, 1908}

Hospederos. Aleyrodidae. En México fue criado de Trialeurodes floridensis (Quaintance) y Paraleyrodes spp. (Myartseva y Varela Fuentes 2005).

Distribución. Cuba, Haití, Hawai, Puerto Rico, EUA (Noyes 2006); México - Chiapas*, Guanajuato, Nuevo León, San Luis Potosí, Tamaulipas (colección de la UAT).

Material examinado. México: Chiapas, Tapachula, ex Aleyrodidae en Psidium guajava, 89.X.2006, 2 hembras (col. S. Myartseva).

Comentarios. Schauff et al. (1996) registró la distribución de Encarsia variegata para México sin indicación de localidades. Durante el periodo del 2005-2006 esta especie de Aleyrodidae fue criada en 4 estados de México (Myartseva y Varela Fuentes 2005).

\section{AGRADECIMIENTOS}

Los autores agradecen a los entomólogos, quienes colectaron materiales interesantes de Aphelinidae en los estados de Chiapas y Jalisco, México. Agradecemos también al Dr. G.A. Evans (USDA/APHIS/PPQ, c/o Systematic Entomology Laboratory USDA/ARS/BARCWest, Beltsville, Maryland, USA) por el envío de valiosas publicaciones sobre Aphelinidae. El primer autor agradece al Dr. D.R. Kasparyan (Zoological Institute of Academy of Sciences of Russia, Sanct Petersburg, Russia) por su amigable ayuda y asesoría en este trabajo. Gracias también por el apoyo financiero de este trabajo al Departamento de Estudios de Postgrado e Investigación, UAM Agronomía y Ciencias, Universidad Autónoma de Tamaulipas, Cd. Victoria, y a la Facultad de Ciencias Biológicas, Universidad Autónoma de Nuevo León, Monterrey.

\section{LITERATURA CITADA}

Alvarado-Mejía, G. y A. González-Hernández. 1990. Taxonomía de las especies de Aphytis Howard (Hymenoptera: Aphelinidae) y otros géneros en el área citrícola de Nuevo León. Biotam 2 (3): 42-51. 
Anónimo. 1979. Lista de insectos entomófagos de interés agrícola en México. Fitófilo 32 (80): 50-96.

Argov, Y. and Y. Rössler. 1988. Introduction of beneficial insects into Israel for the control of insect pests. Phytoparasitica 16: 303-315.

Babcock,C.S., J.M. Heraty, P.J. De Barro, F. Driver and S. Schmidt. 2001. Preliminary phylogeny of Encarsia Förster (Hymenoptera: Aphelinidae) based on morphology and 28SrDNA. Molecular Phylogenetics and Evolution 18: 306-323.

Barbagallo, S.,S. Longo, I. Patti and C. Rapisarda. 1992. Efficiency of biological control against citrus whiteflies in Italy. Bollettino di Zoologia Agraria e Bachicoltura, Milano 24 (2): 121-135.

Dean, H.A. 1982. Reduced pest status of the Florida red scale on Texas citrus associated with Aphytis holoxanthus. Journal of Economic Entomology 75 (1): 147-149.

De Santis, L. 1979. Catálogo de los himenópteros calcidoideos de América al sur de los Estados Unidos. La Plata, Argentina. 488 pp.

. 1989. Catálogo de los himenópteros calcidoideos (Hymenoptera) al sur de los Estados Unidos. Segundo Suplemento. Acta Entomológica Chilena 15: 9-90.

Ehler, L.E. 1997. Obscure scale declines after parasitic wasp introduced. California Agriculture 51 (6): 29-32.

Evans, G.A. and C.L. Angulo. 1996. A new species of Encarsia (Hymenoptera: Aphelinidae) from Costa Rica. Florida Entomologist 79 (4): 582-586.

Hayat, M. 1989. A revision of the species of Encarsia Foerster (Hymenoptera: Aphelinidae) from India and the adjacent countries. Oriental Insects 23: 1-131.

- 1998. Aphelinidae of India (Hymenoptera: Chalcidoidea): a taxonomic revision. Memoirs on Entomology, International Assoc. Publisher, Gainesville, Florida, USA, 13: $1-416$.

Hennessey, R.D., M.C. Arredondo-Bernal y L.A. Rodríguez del Bosque. 1995. Distribución geográfica y huéspedes alternos de parasitoides afelínidos de Bemisia tabaci (Hymenoptera; Aleyrodidae). Vedalia 2: 61-75.

Huang, J. and A. Polaszek. 1998. A revision of Chinese species of Encarsia Foerster (Hymenoptera: Aphelinidae): parasitoids of whiteflies, scale insects and aphids (Hemiptera: Aleyrodidae, Diaspididae, Aphidoidea). Journal of Natural History 32: 1825-1966.

Jiménez-Jiménez, E. y H.D. Smith. 1958. El empleo de enemigos naturales para el control de insectos que constituyen plagas agrícolas en la República Mexicana. Fitófilo 11 (21): 5-24.

Mound, L.A., J.H. Martin and A. Polaszek. 1994. The insect fauna of Selaginella (Pteridophyta: Lycopsida), with descriptions of three new species. Journal of Natural History 28: 1403-1415.

Myartseva, S.N. and E. Ruíz Cancino. 2000. Annotated checklist of the Aphelinidae (Hymenoptera: Chalcidoidea) of Mexico. Folia Entomológica Mexicana 109: 7- 33.

Myartseva, S.N., E. Ruíz Cancino y J.M. Coronado Blanco. 2004. Aphelinidae (Hymenoptera), pp. 753-757. En: Llorente Bousquets, J.E., J.J. Morrone, Yáñez Ordóñez \& J. Vargas Fernández (Eds.). Biodiversidad, Taxonomía y Biogeografia de Artrópodos de México: hacia una síntesis de su conocimiento. Volumen IV. México, UNAM, 2004, 790 pp. 
Myartseva, S.N. y J.F. Luna Salas. 2005. Encarsia perplexa Huang y Polaszek, 1998 (Hymenoptera: Chalcidoidea, Aphelinidae) en México y el Sureste de Texas, EUA. Folia Entomológica Mexicana 44 (3): 297-304.

Myartseva, S.N., E. Ruíz-Cancino, S.E. Varela-Fuentes y J.M. Coronado-Blanco. 2006. Especies del género Encarsia (Hymenoptera: Aphelinidae) obtenidas de mosquitas blancas (Homoptera: Aleyrodidae) en México en cítricos y otras plantas. Entomología Mexicana 5 (tomo 2): 1120-1124.

Myartseva, S.N. y S.E. Varela Fuentes. 2005. Encarsia variegata Howard (Hymenoptera: Aphelinidae) - a parasitoid of whiteflies Paraleyrodes spp. (Homoptera: Aleyrodidae) in three citrus-producing states of Mexico. Vedalia 12 (1): 23-31.

Noyes, J.S. 1982. Collecting and preserving chalcid wasps (Hymenoptera: Chalcidoidea). Journal of Natural History 16: 315-334.

.2006. Universal Chalcidoidea Database (Computer version, last updated: June 2006).

Polaszek, A., G.A. Evans and F.D, Bennett. 1992. Encarsia parasitoids of Bemisia tabaci (Hymenoptera: Aphelinidae, Homoptera: Aleyrodidae): a preliminary guide to identification. Bulletin of Entomological Research 82: 375-392.

Rivnay, T. and D. Gerling. 1988. Aphelinidae parasitoids (Hymenoptera: Chalcidoidea) of whiteflies (Hemiptera: Aleyrodidae) in Israel, with descriptions of three new species. Entomophaga 32 (5): 463-475.

Schauff, M.E., G.A. Evans and J.M. Heraty. 1996. A pictorial guide to the species of Encarsia (Hymenoptera: Aphelinidae) parasitic on whiteflies (Homoptera: Aleyrodidae) in North America. Proceedings of the Entomological Society of Washington 98 (1): 1-35.

Viggiani, G. 1989. Notes on some Nearctic and Neotropical Encarsia Förster (Hymenoptera: Aphelinidae). Bollettino del Laboratorio di Entomologia Agraria Filippo Silvestri di Portici 46: 207-213. 
